\title{
GEOPHYSICS
}

\section{Noise Reduction with Reflection Supervirtual Interferometry}

\begin{tabular}{|r|l|}
\hline Journal: & Geophysics \\
\hline Manuscript ID & GEO-2019-0571.R1 \\
\hline Manuscript Type: & Technical Paper \\
\hline Keywords: & interferometry, reflection \\
\hline Area of Expertise: & Signal Processing, Seismic Interferometry \\
\hline \multicolumn{2}{l}{} \\
\end{tabular}

\section{SCHOLARONE ${ }^{m}$ \\ Manuscripts}




\title{
Noise Reduction with Reflection Supervirtual Interferometry
}

\author{
Kai Lu ${ }^{1}$, Zhaolun Liu ${ }^{1}$, Sherif Hanafy ${ }^{2}$ and Gerard Schuster ${ }^{1}$ \\ ${ }^{1}$ King Abdullah University of Science and Technology, Thuwal, Saudi Arabia. E-mail: \\ kai.lu@kaust.edu.sa; zhaolun.liu@kaust.edu.sa; gerard.schuster@kaust.edu.sa. \\ ${ }^{2}$ King Fahd University of Petroleum and Minerals, Dhahran, Saudi Arabia. E-mail: \\ sherif.geo@gmail.com.
}

(January 4, 2020)

Running head: Reflection $\boldsymbol{S} \boldsymbol{V I}$

\begin{abstract}
To image deeper portions of the earth, geophysicists must record reflection data with much greater source-receiver offsets. The problem with these data is that the signal-to-noise ratio (SNR) significantly diminishes with greater offset. In many cases, the poor SNR makes the far-offset reflections imperceptible on the shot records. To mitigate this problem, we develop supervirtual reflection interferometry (SVI), which can be applied to far-offset reflections to significantly increase their SNR. The key idea is to select the common pair gathers where the phases of the correlated reflection arrivals differ from one another by no more than a quarter of a period so that the traces can be coherently stacked. The traces are correlated and summed together to create traces with virtual reflections, which in turn are convolved with one another and stacked to give the reflection traces with much stronger SNRs. This is similar to refraction SVI except far-offset reflections are used instead of refractions. The theory is validated with synthetic tests where SVI is applied to far-offset reflection arrivals to significantly improve their SNR. Reflection SVI is also applied to a field dataset where
\end{abstract}

(C) 2020 Society of Exploration Geophysicists. 
the reflections are too noisy to be clearly visible in the traces. After the implementation of reflection SVI, the NMO velocity can be accurately picked from the SVI-improved data, leading to a successful poststack migration for this dataset. 


\section{INTRODUCTION}

To image deeper portions of the earth, geophysicists must record reflection data with much greater source-receiver offsets. One of the reasons for this is that deeper targets lie below salt in the Gulf of Mexico (Mandroux et al., 2013) and offshore Brazil (Morgan et al., 2013). These economic targets can be best characterized by full waveform inversion methods if the diving waves reach this deep. Unfortunately, the rule-of-thumb for recording diving waves that the recorded traces must achieve an offset-to-depth ratio of greater than 5:1, where depth is that of the target. Thus, offsets of $20 \mathrm{~km}$ or more are required. This is a problem because the SNR significantly diminishes with increasing offset, especially for reflections from deep targets. If their SNR can be enhanced, then the deep reflections can be used to enhance the accuracy of reflection full waveform inversion (Chen et al., 2019) for deep targets.

To mitigate this problem we develop supervirtual reflection interferometry (SVI). As an example of far-offset reflections with poor SNR, Figure 1 shows that the refractions and reflections are barely visible after an offset larger than $0.5 \mathrm{~km}$, while they are completely invisible within the cone of surface-wave arrivals denoted by the yellow quadrilateral. Can the far-offset reflection arrivals be enhanced without resorting to stronger and more expensive sources? For refractions, the affirmative answer to this question is to apply super-virtual interferometry (SVI) (Mallinson et al., 2011; Bharadwaj et al., 2012) to the refraction arrivals. The refraction SVI procedure is designed to increase the signal-to-noise ratio (SNR) of far-offset refractions by more than an order-of-magnitude.

The theory of refraction SVI is described in Bharadwaj et al. (2013) and Appendix A, and the key idea is illustrated in Figure 2. Here, there are two steps: the first step is to take 
pairs of geophones that record traces that contain post-critical refractions from the same refractor; these traces are generated from the same source. Correlating one trace with the other gives the correlated trace in Figure 2c. This process can be repeated for the same pair of geophones except for a different source. The result is a correlated trace with a virtual refraction that arrives at the same time as the previously correlated trace. These traces can be stacked together to get a stacked trace with the SNR of the virtual refraction enhanced by the factor $\sqrt{N}$, where $N$ is the number of stacked correlated traces or number of sources. This procedure can be repeated again except we use the convolution operation described in Figure 2d-2f (see Appendix A and Bharadwaj et al. (2013)) to provide traces with virtual refractions with their SNR enhanced by a factor of roughly $N$.

SVI has been successfully applied to many seismic datasets (Hanafy et al., 2011; Bharadwaj et al., 2013; Vaezi and De Meersman, 2014; Li et al., 2018), including 3D refraction data (Edigbue and Al-Shuhail, 2016; Lu and Chávez-Pérez, 2019). SVI can also be applied to diffraction events to increase their SNR (Dai et al., 2011). Despite the success of SVI, it has not been developed for reflections. The reason is that, in the correlation step, the phases of the arrivals in the common pair gather (CPG) (Dong et al., 2006) vary with the source-receiver offset (see Figure 3) because the reflection arrivals have no common raypaths. However, Figure 3b shows that the events are almost aligned in the right half of the picture where the traces have large source-receiver offsets; equivalently, their associated rays almost coincide. Therefore, stacking these traces produces an acceptably small error as shown in Figure 4b. This suggests that SVI can be applied to the far-offset reflections to enhance their SNRs as long as the reflection events to be stacked after the correlation step arrive within a quarter of a period of one another. Indeed, our paper shows that SVI can significantly enhance the SNR of far-offset reflections. 
This paper presents both the theory of reflection SVI and numerical examples that validate its effectiveness. In the theory section, we provide the theoretical justification for applying SVI to far-offset reflections, followed by a quantitative analysis of the source stacking zone and the workflow for reflection SVI. In the numerical results section, reflection SVI is first tested on synthetic data. Then, we apply reflection SVI to a noisy dataset from Africa. Finally, the limitations and applications of reflection SVI are discussed followed by the conclusions.

\section{THEORY}

SVI can be applied to reflection data because the correlated reflections in a pair of far-offset traces have nearly the same traveltimes for a wide range of far-offset sources. This satisfies the source stationarity property for SVI. The stationarity of far-offset reflection sources can be mathematically proven with a two-layer model as shown in Figure 5, assuming that the sources and receivers are along the same line at the surface, reflections are from the same horizontal reflector and the velocity is homogeneous. Here, the traveltime $t\left(x_{s}, x_{r}\right)$ of a far-offset reflection arrival is given by

$$
\begin{aligned}
t\left(x_{s}, x_{r}\right) & =\frac{\left|x_{s}-x_{r}\right|}{v} \sqrt{1+\left(\frac{2 z}{\left|x_{s}-x_{r}\right|}\right)^{2}}, \\
& \approx \frac{\left|x_{s}-x_{r}\right|}{v}\left(1+\frac{2 z^{2}}{\left|x_{s}-x_{r}\right|^{2}}\right)
\end{aligned}
$$

where $x_{s}$ and $x_{r}$ are the source and receiver coordinates, respectively, $z>0$ is the depth of the layer interface and $v$ is the velocity of the first layer. Here we assume the far-offset approximation $\left|x_{r}-x_{s}\right| \gg z$. The arrival time $\tau\left(x_{s}, x_{r}, \Delta x_{r}\right)$ of an event in a correlogram is equal to the time difference of two reflection arrivals that are excited by the same source 
and recorded by a pair of receivers, which, according to equation 1, can be expressed as

$$
\begin{aligned}
\tau\left(x_{s}, x_{r}, \Delta x_{r}\right) & =t\left(x_{s}, x_{r}+\Delta x_{r}\right)-t\left(x_{s}, x_{r}\right), \\
& \approx \frac{\Delta x_{r}}{v}\left(1-\frac{2 z^{2}}{\left|x_{s}-x_{r}\right|\left|x_{s}-x_{r}-\Delta x_{r}\right|}\right), \\
& \approx \frac{\Delta x_{r}}{v}\left(1-\frac{2 z^{2}}{\left|x_{s}-x_{r}\right|^{2}}\right),
\end{aligned}
$$

where $\Delta x_{r}$ is the separation of the receiver pair $\mathbf{R}_{\mathbf{1}}$ and $\mathbf{R}_{\mathbf{2}}$ as shown in Figure 5 and we impose the small receiver-separation constraint $\Delta x_{r} \ll\left|x_{s}-x_{r}\right|$. We now define a common pair gather as a collection of correlograms. In order to study the phase variation of the correlated events in the common pair gather (see Figure 3b), we calculate the time difference of two neighboring correlated arrivals with source separation $\Delta x_{s}$ by using equation 2 :

$$
\begin{aligned}
\Delta \tau & =\left|\tau\left(x_{s}+\Delta x_{s}, x_{r}, \Delta x_{r}\right)-\tau\left(x_{s}, x_{r}, \Delta x_{r}\right)\right|, \\
& \approx\left|\frac{\partial \tau\left(x_{s}, x_{r}, \Delta x_{r}\right)}{\partial x_{s}}\right| \Delta x_{s}, \\
& =\frac{T_{0}}{4} \frac{16 z^{2} \Delta x_{r}}{\left(x_{s}-x_{r}\right)^{3}} \frac{\Delta x_{s}}{\lambda} .
\end{aligned}
$$

Here $\lambda$ is the dominant wavelength, and $T_{0}=\lambda / v$ is the dominant period. If $O\left(\Delta x_{s}\right) \leq$ $O(\lambda)^{1}$, which is true assuming the source distribution is not extremely coarse in the survey, we can conclude that

$$
|\Delta \tau| \ll \frac{T_{0}}{4}
$$

where we assume that $\Delta x_{r} \ll\left|x_{s}-x_{r}\right|$ and $z \ll\left|x_{s}-x_{r}\right|$. Equation 4 says that the correlated reflection events in two neighboring far-offset traces arrive within a time much less than a quarter of a period. Therefore, stacking the correlograms associated with the same pair of receivers in a common pair gather $(\mathrm{CPG})$ will significantly improve the SNR

\footnotetext{
${ }^{1} \lambda$ is usually around one hundred meters to several hundred meters.
} 
of the stacked reflection arrival.

Now we introduce a concept of the source stacking zone by imposing the stationary source condition

$$
\left|\tau_{1}-\tau_{2}\right|<\frac{T_{0}}{4}
$$

where $\tau_{1}$ and $\tau_{2}$ are the traveltimes of the events in the correlogram for the sources at locations $\mathbf{S}_{\mathbf{1}}$ and $\mathbf{S}_{\mathbf{2}}$, respectively. The locations $\mathbf{S}_{\mathbf{1}}$ and $\mathbf{S}_{\mathbf{2}}$ that satisfy the above condition define the region of the source stacking zone illustrated in Figure 5. Only the correlograms associated with sources within the source stacking zone are to be stacked.

In the following we introduce the implementation of the correlation step in reflection SVI with the concept of the source stacking zone. Unlike refraction SVI, where the formula of the stacked trace (equation 10) is not a function of the source location, the formula of the stacked trace in reflection SVI is a function of the source location. The reason is that the phases of the virtual reflections in the correlograms vary with source locations (see Figure $3 \mathrm{~b})$, so the strengthened virtual reflections in the stacked trace should also have varying phases. The formula for the stacked virtual reflection trace in reflection SVI is given by

$$
d\left(t, \mathbf{R}_{\mathbf{1}}, \mathbf{R}_{\mathbf{2}}, \mathbf{S}_{\mathbf{0}}\right)^{v i r t}=\sum_{\mathbf{S} \in \Gamma\left(\mathbf{R}_{\mathbf{1}}, \mathbf{R}_{\mathbf{2}}, \mathbf{S}_{\mathbf{0}}\right)} d\left(t, \mathbf{R}_{\mathbf{2}}, \mathbf{S}\right) \otimes d\left(t, \mathbf{R}_{\mathbf{1}}, \mathbf{S}\right),
$$

where $\mathbf{S}_{\mathbf{0}}$ is the source associated with the virtual reflection trace to be strengthened, $\otimes$ is the cross-correlation operation and $\Gamma\left(\mathbf{A}, \mathbf{B}, \mathbf{S}_{\mathbf{0}}\right)$ is the source stacking zone associated with the given source and receiver locations. Assuming that $\mathbf{S}_{\mathbf{0}}$ is located at the center of the source stacking zone, according to equation 5 , the distance $d r$ from $\mathbf{S}_{\mathbf{0}}$ to the source 
stacking zone boundaries can be approximately calculated by solving the equation

$$
2 d r \cdot\left|\frac{\partial \tau\left(x_{s}, x_{r}, \Delta x_{r}\right)}{\partial x_{s}}\right|_{x_{s}=x_{s_{0}}} \mid=\frac{T_{0}}{4}
$$

where $x_{s_{0}}$ is the coordinate of source $\mathbf{S}_{\mathbf{0}}$. Using equation 3 , the solution is given by

$$
d r=\frac{T_{0} v\left|x_{s_{0}}-x_{r}\right|^{3}}{32 \Delta x_{r} z^{2}}
$$

where equation 8 determines the selection of the traces to be correlated and stacked together in reflection SVI. In practice, the velocity model is often not accurately known, and it can be more complex than the layered model in Figure 5. In this case we can use the average velocity as the value of $v$. The source stacking zone is somewhat similar to the end-fire zone defined for surface-wave interferometry (Campillo and Roux, 2015) and refraction SVI (Lu and Chávez-Pérez, 2019), and an illustration of it for 3D surveys is shown in Figure 6.

The convolution step in reflection SVI can be represented as

$$
d\left(t, \mathbf{R}_{\mathbf{2}}, \mathbf{S}_{\mathbf{0}}\right)^{s v i}=\sum_{\mathbf{R}_{\mathbf{1}} \in \hat{\Gamma}\left(\mathbf{R}_{\mathbf{2}}, \mathbf{S}_{\mathbf{0}}\right)} d\left(t, \mathbf{R}_{\mathbf{1}}, \mathbf{S}_{\mathbf{0}}\right) * d\left(t, \mathbf{R}_{\mathbf{1}}, \mathbf{R}_{\mathbf{2}}, \mathbf{S}_{\mathbf{0}}\right)^{v i r t}
$$

where $d\left(t, \mathbf{R}_{\mathbf{2}}, \mathbf{S}_{\mathbf{0}}\right)^{s v i}$ represents the trace containing the reflection arrival associated with the receiver at $\mathbf{R}_{2}$ and the source at $\mathbf{S}_{\mathbf{0}}$ with an improved SNR, and $*$ represents the convolution operation. Here, $\hat{\Gamma}\left(\mathbf{R}_{\mathbf{2}}, \mathbf{S}_{\mathbf{0}}\right)$ is the range of points that are between $\mathbf{S}_{\mathbf{0}}$ and $\mathbf{R}_{\mathbf{2}}$ and satisfy the far-offset condition and small receiver-separation condition for the given receiver at $\mathbf{R}_{\mathbf{2}}$ and source at $\mathbf{S}_{\mathbf{0}}$. Assuming $d\left(t, \mathbf{R}_{\mathbf{1}}, \mathbf{R}_{\mathbf{2}}, \mathbf{S}_{\mathbf{0}}\right)^{\text {virt }}$ has the correct phase, each term in the summation in equation 9 has constant phase that is equivalent to the phase of the true far-offset reflection arrivals associated with the receiver at $\mathbf{R}_{\mathbf{2}}$ and the source at 
$\mathbf{S}_{\mathbf{0}}$, so equation 9 results in coherent stacking of events, and the stacking after convolution does not suffer the non-stationary phase problem as the correlation step does. Therefore, there is no need to define a stacking zone for receivers in the convolution step.

The workflow of reflection SVI is illustrated in Figure 7, which can be summarized as

1. Window around the reflection events of interest. The temporal width of the window can be several periods.

2. Calculate the source stacking zone for each source and receiver pair combination following equation 8 .

3. Create virtual traces by calculating the correlograms and stacking traces for sources in the stacking zone following equation 6.

4. Create supervirtual far-offset reflections by convolution and stacking using equation 9.

\section{NUMERICAL RESULTS}

We now first test the effectiveness of reflection SVI on synthetic data and then apply it on field data recorded in Olduvai Gorge, Tanzania.

\section{Synthetic Example}

Reflection SVI is tested on a synthetic dataset with the velocity and the reflectivity models shown in Figure 8. The survey consists of 100 sources and 200 receivers evenly distributed along a 2-km survey line, with a source spacing of $20 \mathrm{~m}$ and a receiver spacing of $10 \mathrm{~m}$. The synthetic data are generated by Born modeling, containing only primaries. A shot gather 
example with the source located at $X=1.4 \mathrm{~km}$ is shown in Figure 9a. The source wavelet is a Ricker wavelet with a peak frequency of $10 \mathrm{~Hz}$. Strong random noise is added to the synthetic data to generate the noisy data that are to be improved by reflection SVI as shown in 9b. Reflection SVI is applied to reflection arrivals from two reflectors shown in Figure 8b, and the corresponding arrivals in the data are marked in Figure 9a. Figure 9c presents the far-offset data with the two events after SVI processing, where the reflection arrivals are recovered with the correct phases, and their SNRs are significantly enhanced compared to the noisy data. Figure 10 compares the normalized traces extracted from the synthetic data, noisy data and SVI data at $X=100 \mathrm{~m}$ and $X=300 \mathrm{~m}$, respectively. In both comparisons, the reflection arrivals in the SVI data closely agree with those in the synthetic data in terms of the phase, and the noise is significantly suppressed. It demonstrates that SVI significantly improves the SNR of reflections and conserves the accurate phase information.

\section{Field Example}

We now apply reflection SVI to a land dataset recorded over the Olduvai Basin, Tanzania. The purpose of the seismic survey is to delineate the interface between the bedrock of the basin and the overlying sediments, which will be denoted as the bedrock interface. The survey geometry is displayed in Figure 11. The first profile of this survey consists of 240 vertical-component geophones deployed along a $1.2-\mathrm{km}$ line, with a geophone spacing of 5 $\mathrm{m}$, and 120 shots fired with a spacing of $10 \mathrm{~m}$. A 90-kg accelerated weight drop was used as the seismic source, and the peak frequency of the source wavelet is $15 \mathrm{~Hz}$. The recorded data are noisy because of the scattering in the near-surface unconsolidated sediments and the 4.5-9.0 m/s wind noise. Band-pass filtering and AGC are applied to the data and an example of a shot gather with the source located at $1.0 \mathrm{~km}$ after processing is given in 
Figure 12a, where the reflections are almost invisible for $0<X<500 \mathrm{~m}$.

We now attempt to image the bedrock reflector by poststack migration of the recorded data. The processing workflow includes windowing the reflection events associated with this reflector from the shot gather, sorting the picked data to the CDP gather, applying normal moveout (NMO) correction to flatten the events and stacking the NMO-corrected traces to get a stacked reflection time section (Sheriff and Geldart, 1995). However, the noisy data shown in Figure 12a are difficult to process by standard methods because the near-offset reflections are severely contaminated by strong surface waves and the far-offset reflections suffer from low SNR. This means identification of the targeted reflection arrivals and picking the correct NMO velocities are almost impossible.

Reflection SVI is applied to improve the SNR of the far-offset reflections. The input traces are windowed along the estimated reflection events ${ }^{2}$ and the SVI result is shown in Figure 12b, where the SNR of the far-offset reflection is significantly improved. The estimated velocity $v$ and depth $z$ used to calculate the source stacking zone window times are obtained from the inverted traveltime tomogram (Lu et al., 2019). Reflection data before and after SVI are both sorted into the CDP domain and are compared in Figure 13. The reflection events are now clearly visible in the data after SVI processing, allowing for the estimation of the correct stacking velocity for NMO correction. This is crucial for accurate reflection imaging especially when the near-offset reflections are not identifiable. The NMO velocity can now be picked from the SVI data (the velocity from the raw data is unpickable in the velocity semblance image), the raw and SVI reflections can be flattened and stacked to get the zero-offset time sections in Figure 14. The SVI stacked reflections show much better

\footnotetext{
${ }^{2}$ The tomogram revealed the approximate depth of the bedrock and the velocity distribution, so the wide time window that possibly contained the bedrock reflector can be estimated.
} 
continuity and have higher SNR than the reflections in the raw data. The traveltimes of the raw and SVI stacked reflection sections in Figure 14 are consistent with one another, which is an important sanity check demonstrating that reflection SVI is reliable. The stacked SVI reflection traces are migrated to the depth domain by using the velocity from the tomogram. A comparison of the migration images using the raw and SVI data is presented in Figures 15a and 15b, where the image from the SVI data has better coherency. Figure 15c displays the traveltime tomogram. Here, the traveltimes are those picked from the first arrivals in the recorded traces. The geological structure interpreted from the tomogram and the reflection image are consistent with one another, further demonstrating the reliability of the reflection processing. Details about the geological interpretation of this seismic section are given in Lu et al. (2019).

\section{DISCUSSION}

Reflection SVI is similar to data binning (Yilmaz, 2001) in the sense that both methods stack reflection arrivals that arrive within about a quarter of a period to improve their SNR. Selecting traces from the source stacking zone in reflection SVI plays a similar role as sorting traces to a common-cell gather in data binning. The difference is that the stacking operation in SVI is carried out in the CPG domain, where the phase shifts between adjacent traces are smaller ${ }^{3}$. Given a phase-error tolerance, smaller shifts between traces means more traces are available for stacking and better SNR improvement can be achieved. The SNR improvement of reflection SVI is similar to that of refraction SVI, which is at most $\sqrt{M N}$ (Bharadwaj et al., 2012). Here $M$ and $N$ are the number of stacks in the correlation and convolution steps, respectively. However, the stacking of the correlated traces in reflection

\footnotetext{
${ }^{3}$ The traveltimes in CDP gather and common pair gather are presented as $t$ and $\tau$ in equation 3 and 4 , respectively, and $\partial \tau / \partial x_{s}$ is much smaller than $\partial t / \partial x_{s}$.
} 
SVI is not strictly coherent; the energy improvement is slightly decreased. Therefore, the SNR improvement of reflection SVI is somewhat smaller than $\sqrt{M N}$.

Unlike conventional SVI which is a purely data-driven method, reflection SVI requires a rough estimate of the reflector depth and velocity to calculate the source stacking zone (equation 8). Such information can be estimated from well logs, tomograms, or the sections from other surveys. The velocity and depth can also be used to calculate the phase shifts between neighboring traces in the correlogram (equation 3). If the velocity and depth information is accurate, flattening the events in the correlogram by static shifts before stacking is an option to improve the SVI results. Even if the velocity and depth estimates are inaccurate, stacking traces associated with the sources in the stacking zone will tend to cancel the out-of-phase events and reinforce the coherent ones.

A challenge of reflection SVI is that a time window must be defined around the targeted reflection arrivals. Therefore, applying SVI to all reflection arrivals in a complex model will be challenging. However, if the subsurface has a roughly layered structure, the windows for reflection arrivals from each layer can be estimated, and SVI can be applied systematically to all reflections. In our field data case, an important contribution from SVI is that the NMO velocity can be picked after the SVI processing. SVI improved the quality of the stacked section as well, although it is not as significant as the improvement in the data quality. Some other possible applications of reflection SVI include regional passive seismic surveys, where the surface wave energy dominates and the weak reflection arrivals can sometimes be observed in the correlated data (Nakata et al., 2011; Clayton, 2018). Reflection SVI might be able to improve the data quality and help image deep layers such as the Moho.

For near-offset reflection data, the far-offset assumption of reflection SVI $z \ll\left|x_{s}-x_{r}\right|$ is 
violated. However, if the velocity and the reflector depth are well known, we can analytically calculate the shifts between near-offset traces in the CPG and roughly flatten the near offset events so that SVI can be applied.

If the far-offset reflections are post-critical reflections, then there is a phase change in the reflection arrivals that depends on the post-critical reflection angle. This will lead to phase changes in the correlograms in a CPG. If these phase changes are significant, then they must be corrected prior to stacking the traces in a correlogram. This can be accomplished by breaking up the traces in the CMP into subsets of correlograms where there is only a slight phase change in the traces. Stacking the traces in each subset will then be mostly coherent. The traces in the stacked subsets can then be deconvolved to eliminate any phase changes from one subset to the next. In our synthetic tests and field data tests we did not encounter any significant problems with variable phase changes in the CMPs.

\section{CONCLUSIONS}

The theory of the reflection SVI is presented for far-offset reflections with the following conditions: (1) the far-offset condition where the depth of the reflector is much smaller than the source-receiver offsets; and (2) the close-trace condition where the distance between the two receivers of a correlated trace pair is much smaller than the source-receiver offsets. The workflow of reflection SVI is similar to refraction SVI, except that the source stacking zone should be computed for the correlation step; this is somewhat similar to defining the end-fire zones for refraction SVI. The correlated reflection events with sources located in the stacking zone are almost stationary. To properly estimate the source stacking zone and window parameters a good estimate of the reflector depth and background velocity is recommended. 
Tests with synthetic data show that noisy reflection data can be enhanced to improve the SNR by more than order-of-magnitude. In the noisy land data, the far-offset reflection arrivals from the targeted reflector, which are almost invisible in the raw data, become easily seen in the SVI data. This allows for an accurate estimation of the NMO velocity and noticeably improves the quality of the post migration image.

\section{ACKNOWLEDGMENTS}

We thank the sponsors for supporting the Consortium of Subsurface Imaging and Fluid Modeling (CSIM). We also thank KAUST for providing funding by the CRG grant OCRF2014-CRG3-2300. For computer time, this research used the Computing Group and the Supercomputing Laboratory at KAUST.

\section{APPENDIX A: THEORY OF REFRACTION SVI}

The refraction SVI consists of the two steps illustrated in Figure 2:

1. Correlation of the a) refraction trace $d(t, \mathbf{B}, \mathbf{A})$ recorded at $\mathbf{B}$ for a source at $\mathbf{A}$ with the b) refraction trace $d(t, \mathbf{C}, \mathbf{A})$ recorded at $\mathbf{C}$ gives the $\mathrm{c})$ virtual refraction trace $d(t, \mathbf{B}, \mathbf{A}) \otimes d(t, \mathbf{C}, \mathbf{A})$ at $\mathbf{C}$. Here, we assume $\mathbf{C}$ and $\mathbf{B}$ are at post-critical receiver positions for the source at $\mathbf{A}$ and the head waves propagate along the same refracting interface. To increase the SNR, the correlograms $d(t, \mathbf{B}, \mathbf{A}) \otimes d(t, \mathbf{C}, \mathbf{A})$ are summed over the postcritical source positions at $\mathbf{A}$ to give the virtual refraction trace $d(t, \mathbf{C}, \mathbf{B})^{\text {virt }}$ at $\mathbf{C}$ :

$$
d(t, \mathbf{C}, \mathbf{B})^{v i r t}=\sum_{\mathbf{A} \in \Gamma(\mathbf{B}, \mathbf{C})} d(t, \mathbf{B}, \mathbf{A}) \otimes d(t, \mathbf{C}, \mathbf{A})
$$


where $\otimes$ denotes the cross-correlation operation and $\Gamma(\mathbf{B}, \mathbf{C})$ corresponds to the range of far-offset source locations for the refractions to propagate from $\mathbf{A}$ to the receiver pair $\mathbf{B}$ and $\mathbf{C}$.

In the frequency domain, temporal correlation of two traces is equivalent to multiplying the conjugate of one trace's spectrum by the other. This means that for the phase spectrum the a) traveltime $\tau_{\mathbf{A B}}=\tau_{\mathbf{A B}^{\prime}}+\tau_{\mathbf{B}^{\prime} \mathbf{B}}$ is subtracted from the b) traveltime $\tau_{\mathbf{A C}}=\tau_{\mathbf{A B}^{\prime}}+\tau_{\mathbf{B}^{\prime} \mathbf{C}}$ to give the traveltime $\tau_{\mathbf{A C}}-\tau_{\mathbf{A B}}=\tau_{\mathbf{B}^{\prime} \mathbf{C}}-\tau_{\mathbf{B}^{\prime} \mathbf{B}}$. A stationary event in the correlogram appears at the same time $\tau_{\mathbf{A C}}-\tau_{\mathbf{A B}}$ for all post-critical source positions at A. After the correlation of two traces the traveltimes associated with the common raypath $\overrightarrow{\mathbf{A B}^{\prime}}$ in Figure $2 \mathrm{c}$ are eliminated and the remaining traveltime is associated with the traveltime of the solid ray $\overrightarrow{\mathbf{B}^{\prime} \mathbf{C}}$ subtracted from that associated with the red ray $\overrightarrow{\mathbf{B}^{\prime} \mathbf{B}}$.

2. Convolution of the d) virtual refraction trace $d(t, \mathbf{C}, \mathbf{B})^{\text {virt }}$ with the e) trace $d(t, \mathbf{B}, \mathbf{A})$ recorded at $\mathbf{B}$ gives the $\mathrm{f})$ supervirtual refraction trace $d(t, \mathbf{C}, \mathbf{B})^{v i r t} * d(t, \mathbf{B}, \mathbf{A})$. To increase the SNR, the supervirtual traces are summed over the post-critical receiver locations B:

$$
d(t, \mathbf{C}, \mathbf{A})^{s v i}=\sum_{\mathbf{B} \in \hat{\Gamma}(\mathbf{C}, \mathbf{A})} d(t, \mathbf{C}, \mathbf{B})^{v i r t} * d(t, \mathbf{B}, \mathbf{A})
$$

where $*$ denotes the convolution operation and $\hat{\Gamma}(\mathbf{C}, \mathbf{A})$ defines the range of receiver locations $\mathbf{B}$ for far-offset refractions excited at $\mathbf{A}$, and $\mathbf{B}$ is located between $\mathbf{A}$ and C. The traveltimes along the black (red) rays have positive (negative) polarity. 


\section{REFERENCES}

Bharadwaj, P., G. T. Schuster, and I. Mallinson, 2012, Theory of supervirtual refraction interferometry: Geophysical Journal International, 288, 263-273.

Bharadwaj, P., X. Wang, G. Schuster, and K. McIntosh, 2013, Increasing the number and signal-to-noise ratio of OBS traces with supervirtual refraction interferometry and freesurface multiples: Geophysical Journal International, 192, 1070-1084.

Campillo, M., and P. Roux, 2015, Seismic imaging and monitoring with ambient noise correlations: Treatise on geophysics, Second Edition, 1, 391417.

Chen, Y., Z. Feng, L. Fu, A. AlTheyab, S. Feng, and G. Schuster, 2019, Multiscale reflection phase inversion with migration deconvolution: Geophysics, 85, no. 1, R55-R73.

Clayton, R. W., 2018, Imaging the subsurface with ambient noise autocorrelations: SEG technical Program Expanded Abstracts 2018, 4852-4856.

Dai, W., T. Fei, Y. Luo, and G. Schuster, 2011, Supervirtual interferometric diffractions as guide stars: SEG technical Program Expanded Abstracts 2011, 3819-3823.

Dong, S., J. Sheng, and G. Schuster, 2006, Theory and practice of refraction interferometry: SEG technical Program Expanded Abstracts 2006, 3021-3025.

Edigbue, P., and A. Al-Shuhail, 2016, Three-dimensional supervirtual seismic refraction interferometry: Arabian Journal of Geosciences, 9, no. 8, 537.

Hanafy, S. M., O. AlHagan, and F. AlTawash, 2011, Supervirtual refraction interferometry: Field data example over a colluvial wedge: SEG technical Program Expanded Abstracts $2011,3814-3818$.

Li, J., K. Lu, G. Schuster, and S. Hanafy, 2018, Two robust imaging methodologies for challenging environments: Wave-equation dispersion inversion of surface waves and guided waves and supervirtual interferometry+tomography for far-offset refractions: Interpreta- 
tion, 6, no. 4, SM27-SM37.

Lu, K., and S. Chávez-Pérez, 2019, 3D super-virtual refraction interferometry: Geophysics. (accepted subjected to moderate revision).

Lu, K., S. Hanafy, I. Stanistreet, J. Njau, K. Schick, N. Toth, H. Stollhofen, and G. Schuster, 2019, Seismic imaging of the Olduvai Basin, Tanzania: Palaeogeography, Palaeoclimatology, Palaeoecology, 533. (https://doi.org/10.1016/j.palaeo.2019.109246).

Mallinson, I., P. Bharadwaj, G. Schuster, and H. Jakubowicz, 2011, Enhanced refractor imaging by supervirtual interferometry: The Leading Edge, 30, 546-550.

Mandroux, F., B. S. Ong, C.-O. Ting, S. Mothi, T. Huang, and Y. Li, 2013, Broadband, long-offset, full-azimuth, staggered marine acquisition in the Gulf of Mexico: First Break, 31, no. 6, 125-132.

Morgan, J., M. Warner, R. Bell, J. Ashley, D. Barnes, R. Little, K. Roele, and C. Jones, 2013, Next-generation seismic experiments: wide-angle, multi-azimuth, threedimensional, full-waveform inversion: Geophysical Journal International, 195, no. 3, $1657-1678$.

Nakata, N., R. Snieder, T. Tsuji, K. Larner, and T. Matsuoka, 2011, Shear wave imaging from traffic noise using seismic interferometry by cross-coherence: Geophysics, 76, no. 6, SA97-SA106.

Sheriff, R., and L. Geldart, 1995, Exploration seismology: Cambridge University Press.

Vaezi, Y., and K. De Meersman, 2014, Supervirtual S-wave refraction interferometry for converted wave statics and near-surface S-wave velocity model building: 76th EAGE Conference and Exhibition 2014, 2014, 1-5. (doi=10.3997/2214-4609.20140996).

Yilmaz, O., 2001, Seismic data analysis: Society of Exploration Geophysicists. 


\section{LIST OF FIGURES}

1 Shot gather of noisy data where the reflections are barely visible outside the yellow quadrilateral. Within this quadrilateral the energy is dominated by surface wave arrivals.

2 Supervirtual refraction traces are created by correlating post-critical traces and summing over the post-critical sources in (a-c). Here, the red ray in (c) denotes a negative traveltime and the black ray denotes a positive traveltime. In addition, the virtual traces are convolved with the recorded traces and the result is summed over different receiver positions at $\mathbf{B}$ to give the SVI trace in $\mathrm{f}$ ).

3 Common pair gathers (CPGs) of (a) refractions and (b) reflections. Each trace in the CPG is a correlated trace associated with a common receiver pair. The arrivals have constant phases in (a) but varying phases in (b). However, the arrivals in (b) associated with far-offset source locations have the same phase.

4 Comparisons between the reference correlated trace and the stacked trace (a) without and (b) with the source stacking zone. Stacking all traces in the CPG leads to a large phase error, while stacking only the traces whose associated sources are within the source stacking zone results in an acceptable phase error.

5 Diagram of a reflection survey. $\mathbf{R}_{\mathbf{1}}$ and $\mathbf{R}_{\mathbf{2}}$ are receiver locations with a distance of $\Delta x_{r}$, and $\mathbf{S}$ represents the source location with a distance of $x$ to $\mathbf{R}_{\mathbf{1}}$. The reflector is horizontal and its depth is $z$. An illustration of the source stacking zone is plotted, where the sources enjoy the strationarity property.

$6 \quad$ Illustration of the source stacking zone in $3 \mathrm{D}$ survey for a given source at $\mathbf{S}_{\mathbf{0}}$ and the receiver pair at $\mathbf{R}_{1}$ and $\mathbf{R}_{\mathbf{2}}$.

7 The workflow of reflection SVI.

8 The (a) velocity model and (b) reflectivity model for the synthetic test. The ray- 
paths of the two reflections to be processed by SVI are presented in (b).

9 Common shot gathers of (a) the synthetic data, (b) the noisy data and (c) the SVI data. Strong noise is applied to the synthetic data, and the far-offset traces in the noisy data is processed by reflection SVI, resulting in an significant enhancement on the SNR .

10 Comparisons of normalized traces extracted from the synthetic data, the noisy data and the SVI data at $X=100 \mathrm{~m}$ and $X=300 \mathrm{~m}$, respectively. It demonstrates that reflection SVI improves the SNR, and accurately recovers the phase information.

11 The location of the seismic survey in the western Olduvai Basin, Tanzania. (Lu et al., 2019)

12 Common shot gather of (a) the raw data and (b) the SVI result. The red circle marks the far-offset reflections that are significantly improved.

13 The CDP gathers of (a) the raw data and (b) the SVI data. The reflection event can barely be seen in the raw data, but are easily recognized in the SVI data.

14 The stacked time section of (a) raw and (b) SVI data. The stacking velocity is picked using the SVI data for both cases. The SVI data are more coherent and less noisy than the raw data, demonstrating the effectiveness of the SVI method.

15 The migration reflection image in the depth domain from (a) the raw data and (b) the SVI data. The migration image from the SVI data show better coherence and better focused energy than that from the raw data. (c) presents the traveltime tomogram (Lu et al., 2019). The purple solid line and blue dashed line mark the bedrock interface interpreted from the tomogram and the reflection image, respectively. The shape of the bedrock reflector is consistent with that inferred from the tomogram. 


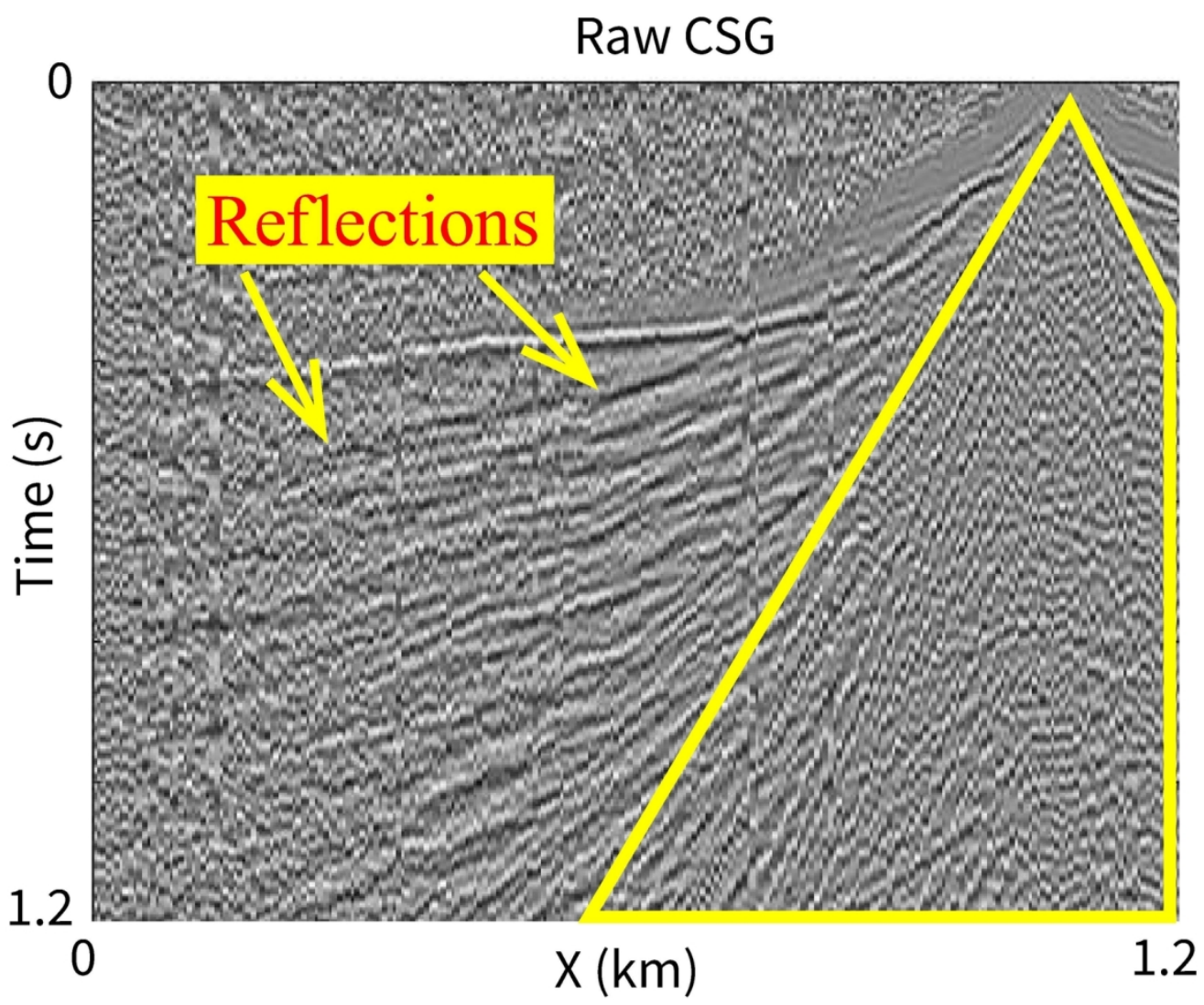

Figure 1: Shot gather of noisy data where the reflections are barely visible outside the yellow quadrilateral. Within this quadrilateral the energy is dominated by surface wave arrivals.

$137 \times 114 \mathrm{~mm}(300 \times 300$ DPI) 
a)

$d(t, B, A)$

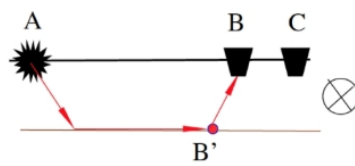

d)

$d(t, C, B)^{v i r t}$

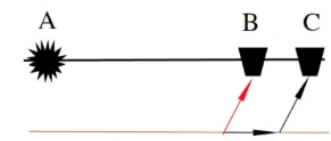

b)

$d(t, C, A)$

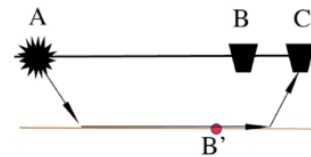

e)

$d(t, B, A)$

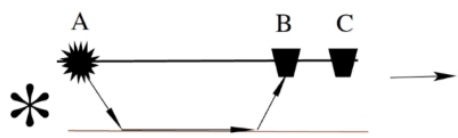

c)

$d(t, C, B)^{\text {virt }}$

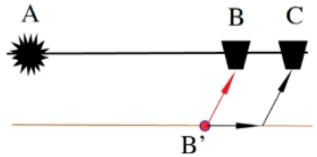

f)

$d(t, C, A)^{s v i}$

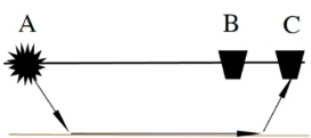

Figure 2: Supervirtual refraction traces are created by correlating post-critical traces and summing over the post-critical sources in (a-c). Here, the red ray in (c) denotes a negative traveltime and the black ray denotes a positive traveltime. In addition, the virtual traces are convolved with the recorded traces and the result is summed over different receiver positions at B to give the SVI trace in f).

$318 \times 147 \mathrm{~mm}$ (300 x 300 DPI) 
(a) CPG of Refractions
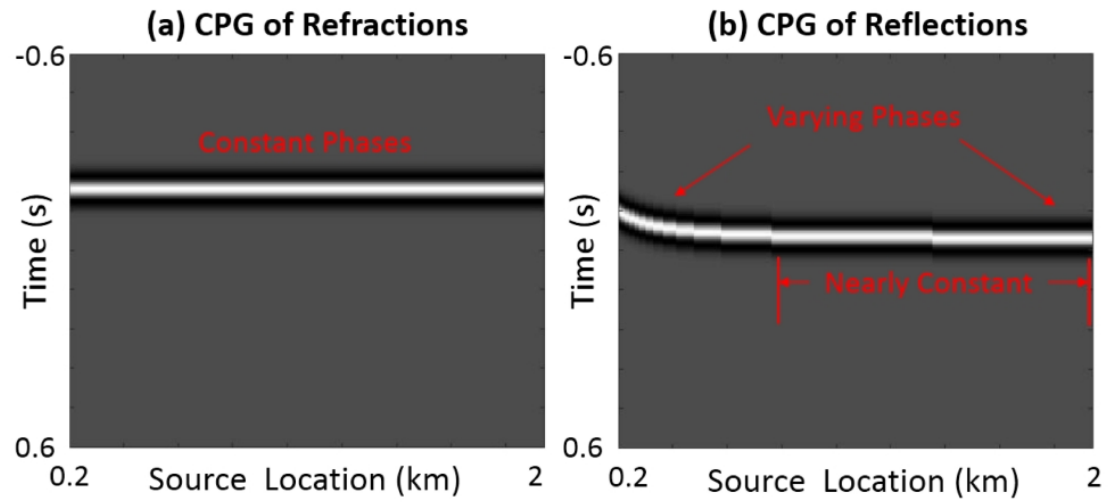

Figure 3: Common pair gathers (CPGs) of (a) refractions and (b) reflections. Each trace in the CPG is a correlated trace associated with a common receiver pair. The arrivals have constant phases in (a) but varying phases in (b). However, the arrivals in (b) associated with far-offset source locations have the same phase.

$266 \times 127 \mathrm{~mm}(300 \times 300 \mathrm{DPI})$ 
(a) Stack without Stacking Zone

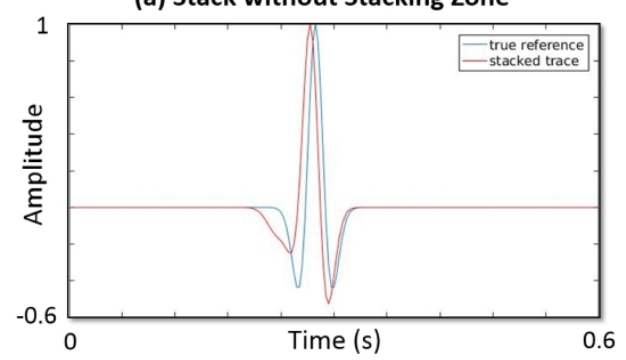

(b) Stack using Stacking Zone

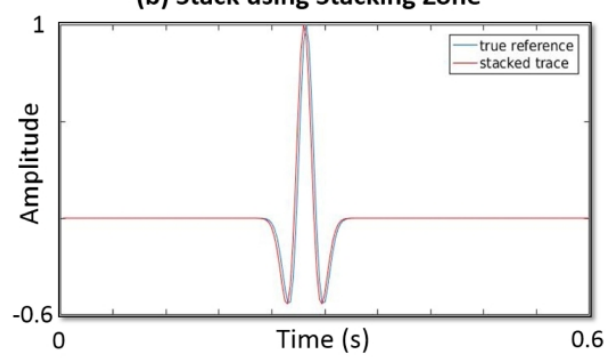

Figure 4: Comparisons between the reference correlated trace and the stacked trace (a) without and (b) with the source stacking zone. Stacking all traces in the CPG leads to a large phase error, while stacking only the traces whose associated sources are within the source stacking zone results in an acceptable phase error.

$342 \times 134 \mathrm{~mm}(300 \times 300 \mathrm{DPI})$ 


\section{Reflection Survey}

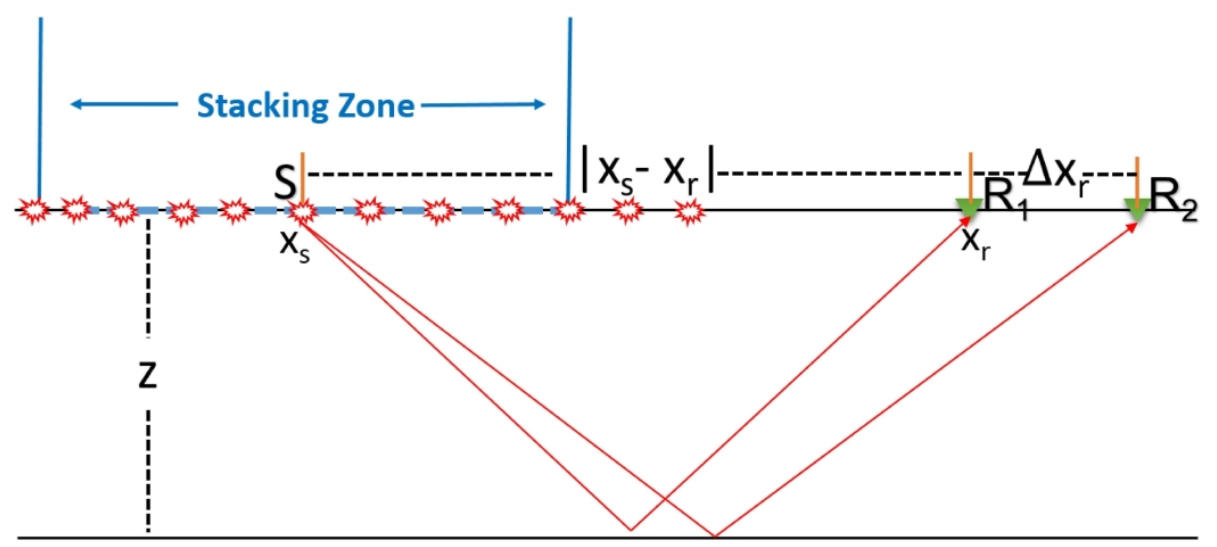

Figure 5: Diagram of a reflection survey. $\mathbf{R}_{1}$ and $\mathbf{R}_{2}$ are receiver locations with a distance of $\Delta x_{r}$, and $\mathbf{S}$ represents the source location with a distance of $x$ to $\mathbf{R}_{1}$. The reflector is horizontal and its depth is $z$. An illustration of the source stacking zone is plotted, where the sources enjoy the strationarity property.

$248 \times 141 \mathrm{~mm}(300 \times 300$ DPI) 


\section{Surface of 3D survey}

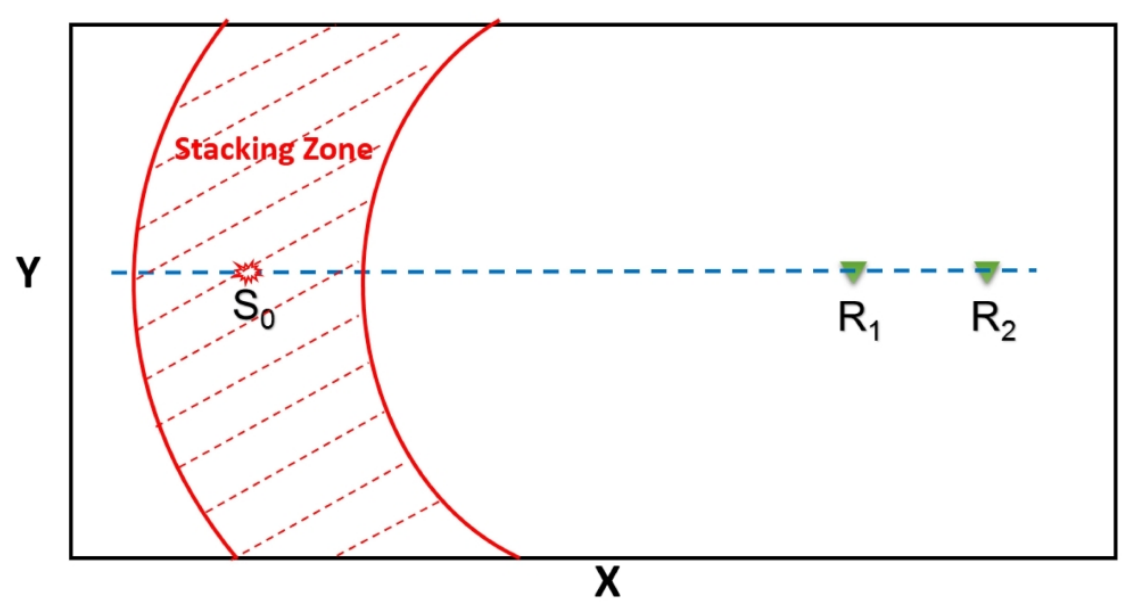

Figure 6: Illustration of the source stacking zone in 3D survey for a given source at $\mathbf{S}_{0}$ and the receiver pair at $\mathbf{R}_{1}$ and $\mathbf{R}_{2}$.

$238 \times 141 \mathrm{~mm}(300 \times 300 \mathrm{DPI})$

This paper presented here as accepted for publication in Geophysics prior to copyediting and composition.

(C) 2020 Society of Exploration Geophysicists. 


\section{Reflection SVI Workflow}

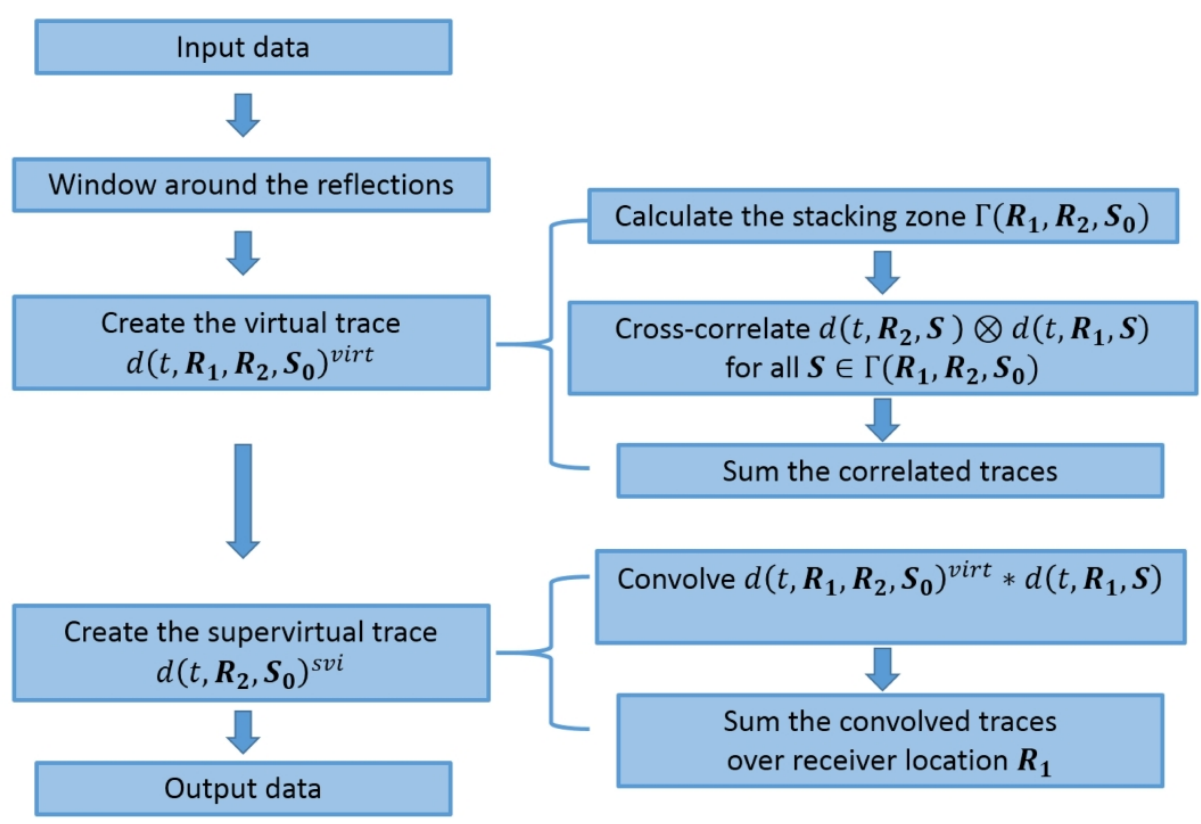

Figure 7: The workflow of reflection SVI.

$277 \times 198 \mathrm{~mm}(300 \times 300 \mathrm{DPI})$ 
(a) Velocity Model

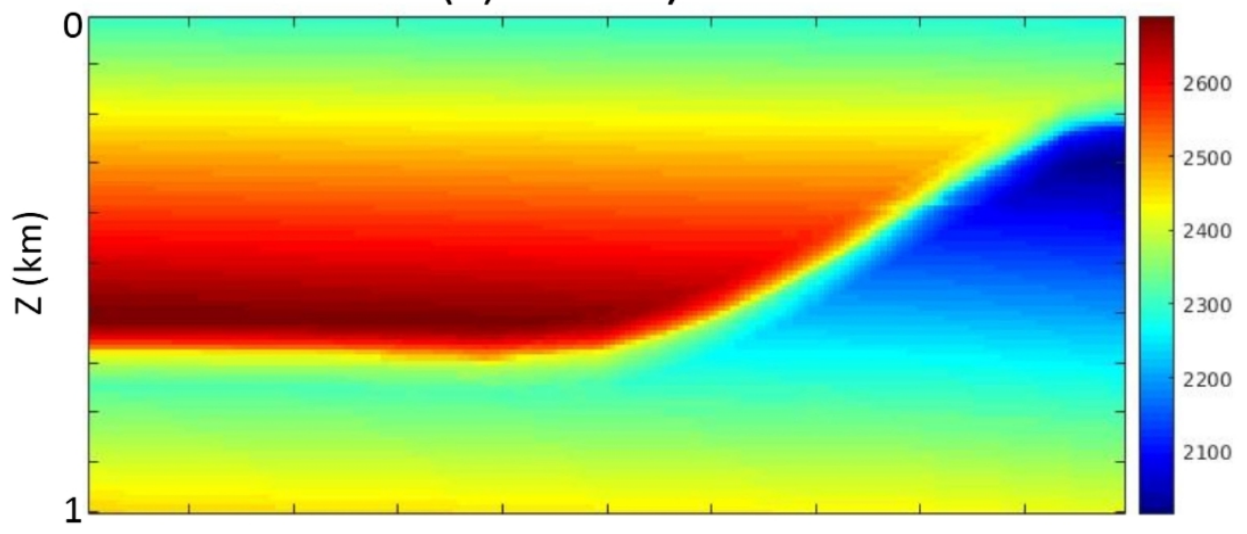

(b) Reflectivity Model

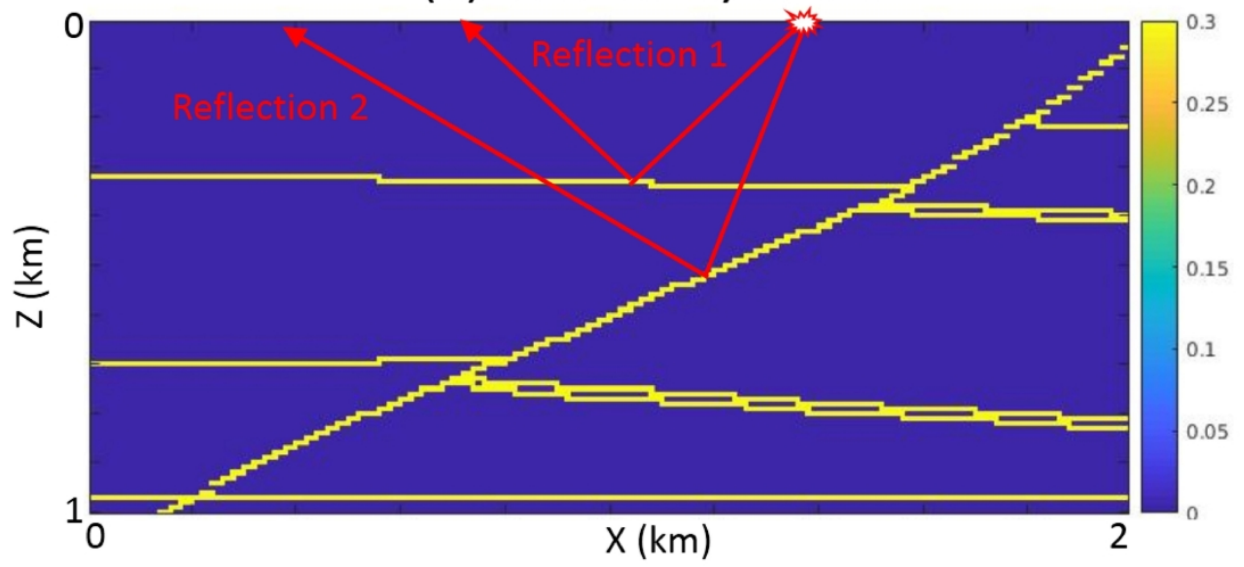

Figure 8: The (a) velocity model and (b) reflectivity model for the synthetic test. The raypaths of the two reflections to be processed by SVI are presented in (b).

$208 \times 196 \mathrm{~mm}(300 \times 300 \mathrm{DPI})$ 
(a) Synthetic Data

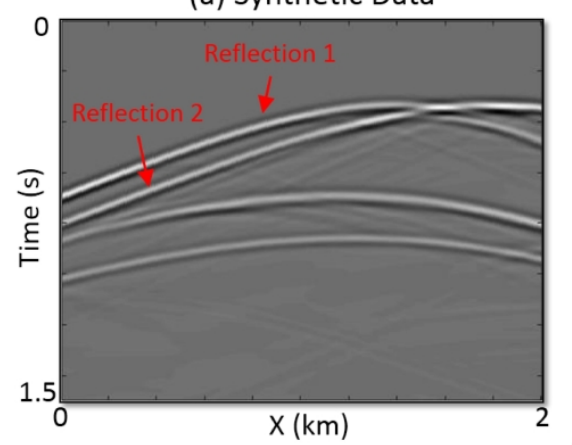

(c) SVI Data (b) Noisy Data

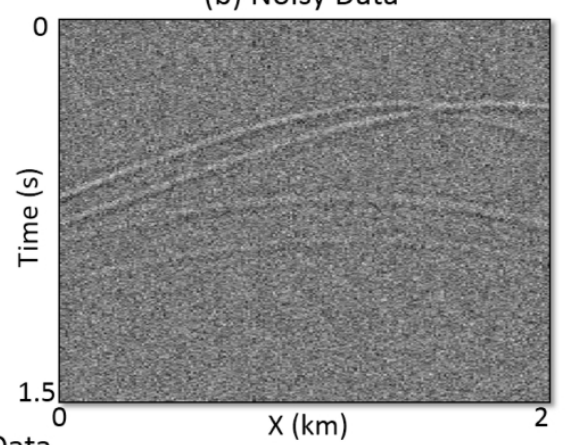

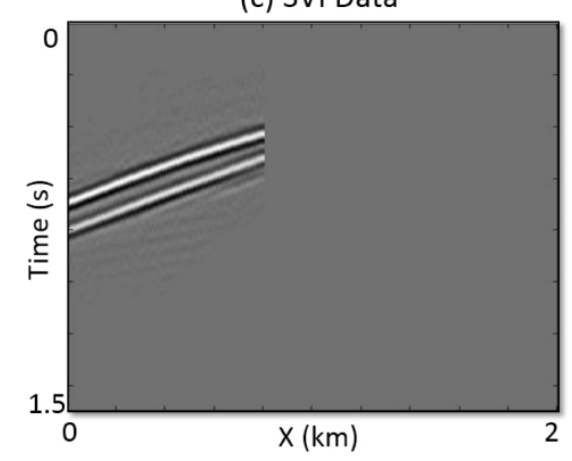

Figure 9: Common shot gathers of (a) the synthetic data, (b) the noisy data and (c) the SVI data. Strong noise is applied to the synthetic data, and the far-offset traces in the noisy data is processed by reflection SVI, resulting in an significant enhancement on the SNR.

$277 \times 196 \mathrm{~mm}(300 \times 300 \mathrm{DPI})$ 
(a) Trace from raw data at $X=100 \mathrm{~m}$

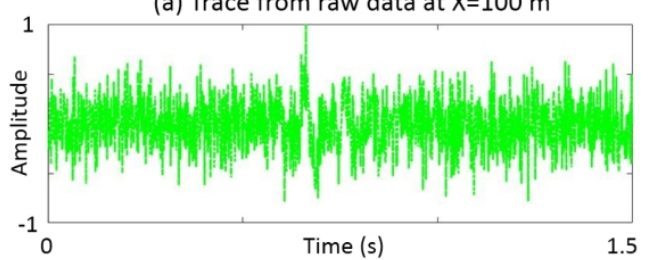

(c) Trace from raw data at $X=300 \mathrm{~m}$

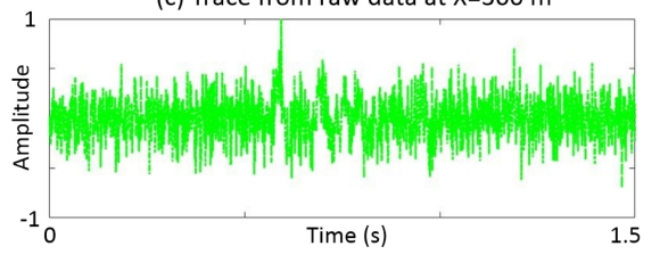

(b) Trace from synthetic and SVI data at X=100 m

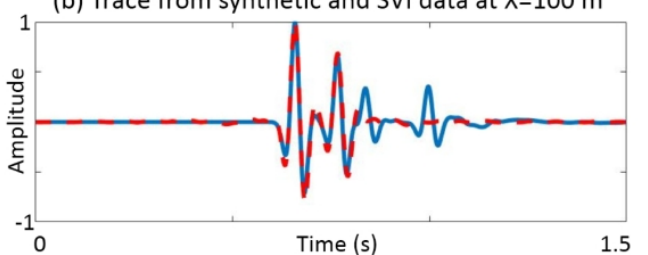

(d) Trace from synthetic and SVI data at X=300 m

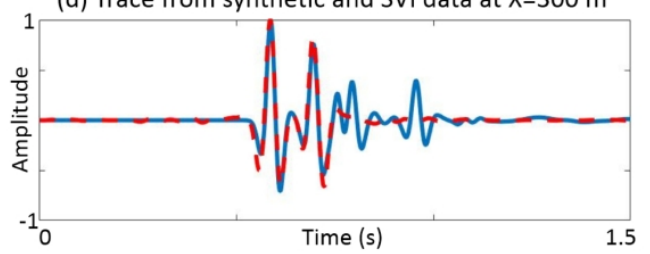

Figure 10: Comparisons of normalized traces extracted from the synthetic data, the noisy data and the SVI data at $\mathrm{X}=100 \mathrm{~m}$ and $\mathrm{X}=300 \mathrm{~m}$, respectively. It demonstrates that reflection SVI improves the SNR, and accurately recovers the phase information.

$340 \times 194 \mathrm{~mm}(300 \times 300$ DPI) 
Seismic Survey in Olduvai Basin

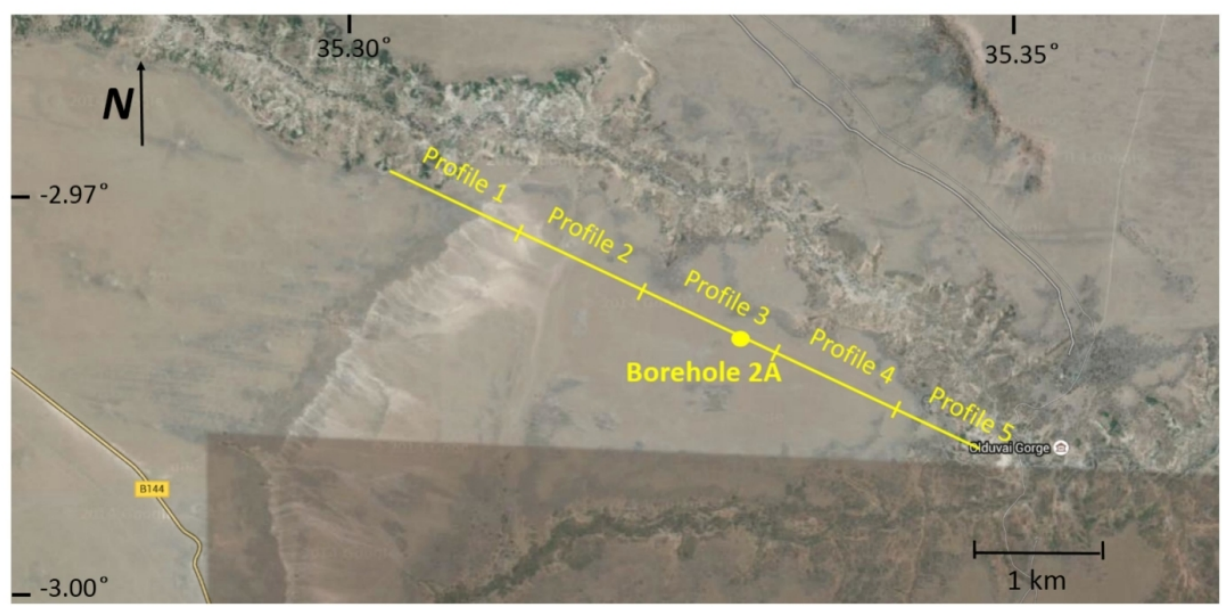

Figure 11: The location of the seismic survey in the western Olduvai Basin, Tanzania. (Lu et al., 2019) $124 \times 70 \mathrm{~mm}(300 \times 300 \mathrm{DPI})$ 
(a) CSG before SVI

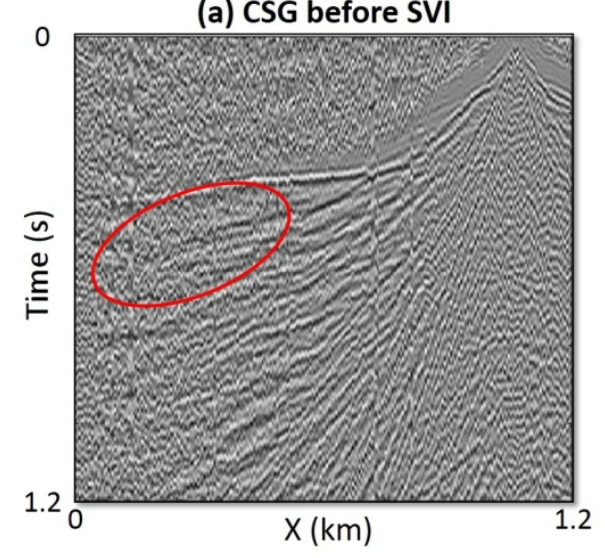

(b) Data after SVI

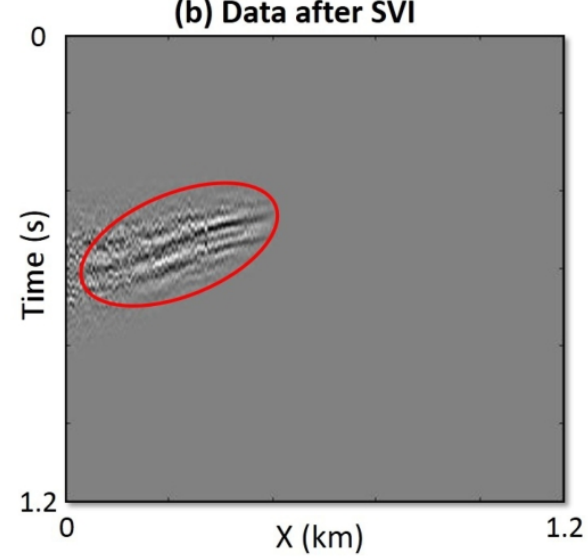

Figure 12: Common shot gather of (a) the raw data and (b) the SVI result. The red circle marks the faroffset reflections that are significantly improved.

$270 \times 123 \mathrm{~mm}(300 \times 300 \mathrm{DPI})$ 

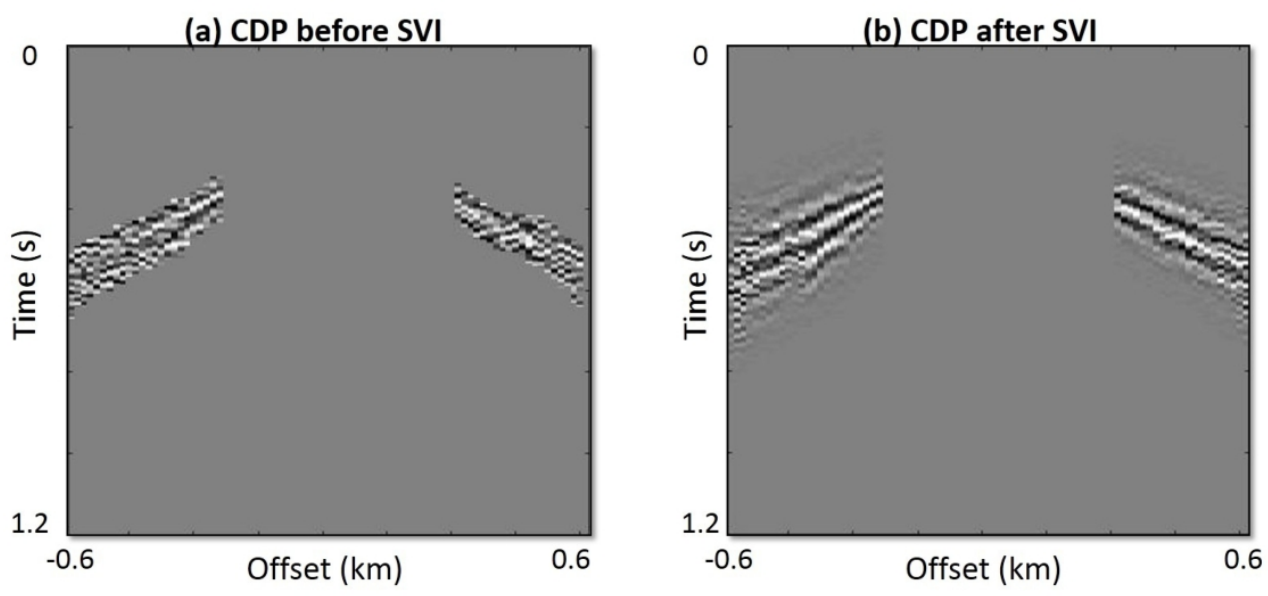

Figure 13: The CDP gathers of (a) the raw data and (b) the SVI data. The reflection event can barely be seen in the raw data, but are easily recognized in the SVI data.

$$
268 \times 125 \mathrm{~mm}(300 \times 300 \mathrm{DPI})
$$


(a) Stacked Time Section with Raw Data

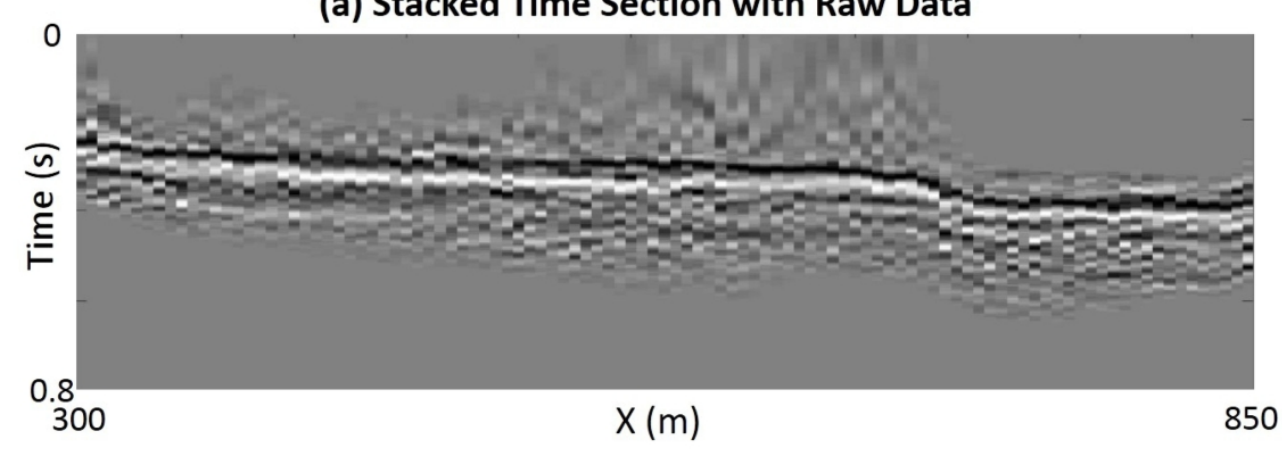

(b) Stacked Time Section with SVI Data

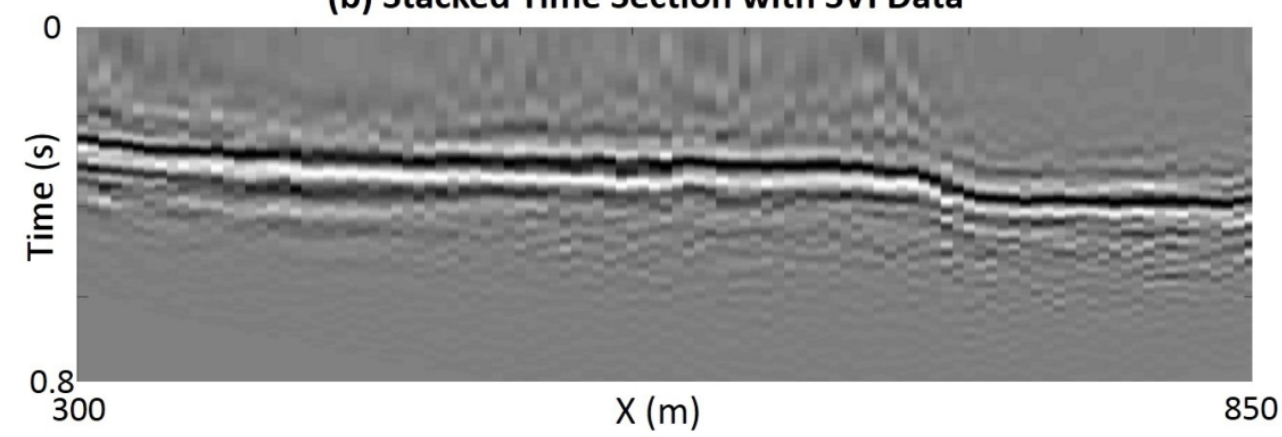

Figure 14: The stacked time section of (a) raw and (b) SVI data. The stacking velocity is picked using the SVI data for both cases. The SVI data are more coherent and less noisy than the raw data, demonstrating the effectiveness of the SVI method.

$230 \times 179 \mathrm{~mm}(300 \times 300 \mathrm{DPI})$ 
(a) Reflection Image in Depth with Raw Data

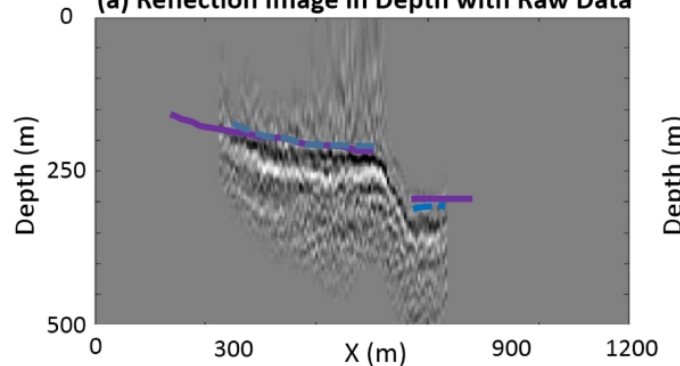

(b) Reflection Image in Depth with SVI Data
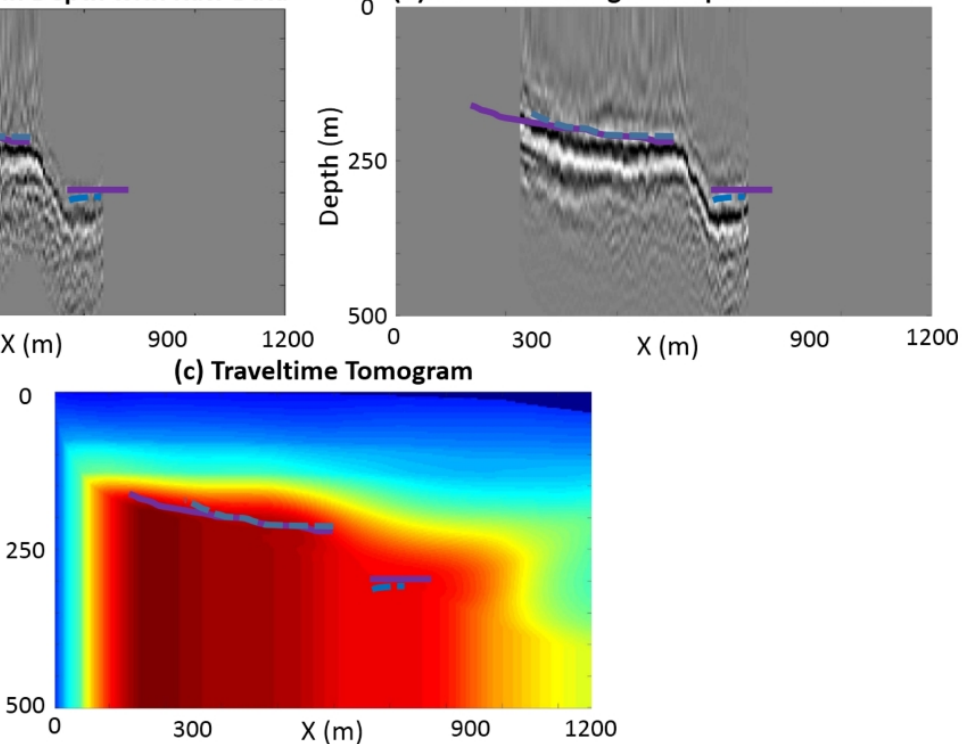

Figure 15: The migration reflection image in the depth domain from (a) the raw data and (b) the SVI data. The migration image from the SVI data show better coherence and better focused energy than that from the raw data. (c) presents the traveltime tomogram (Lu et al., 2019). The purple solid line and blue dashed line mark the bedrock interface interpreted from the tomogram and the reflection image, respectively. The shape of the bedrock reflector is consistent with that inferred from the tomogram.

$$
326 \times 195 \mathrm{~mm}(300 \times 300 \text { DPI) }
$$


DATA AND MATERIALS AVAILABILITY

Data associated with this research are confidential and cannot be released. 